\title{
The Impact of a Community-Based Childhood Obesity Prevention Program on Children's Physical Activity and Fitness
}

\author{
Furong $\mathrm{Xu}^{1, *}$, Mary L. Greaney ${ }^{1}$, Stephanie Marchand ${ }^{2}$, Celeste Corcoran ${ }^{3}$, Heather Di Biasio ${ }^{4}$, \\ Jane Baruch ${ }^{1}$, Deborah Riebe ${ }^{1}$, Geoffrey W. Greene ${ }^{5}$ \\ ${ }^{1}$ Department of Kinesiology, University of Rhode Island, Independence Square II, Kingston, Rhode Island, United States \\ ${ }^{2}$ Kinder Health RI, Wakefield, Rhode Island, United States \\ ${ }^{3}$ Coastal Medical Narragansett Bay Pediatrics, South Kingstown, Rhode Island, United States \\ ${ }^{4}$ Wakefield Pediatrics, Wakefield, Rhode Island, United States \\ ${ }^{5}$ Department of Nutrition and Food Sciences, University of Rhode Island, Fogarty Hall, Kingston, Rhode Island, United States \\ *Corresponding author: fxu2007@uri.edu
}

\begin{abstract}
The purpose of this study is to determine the effects of South County Food, Fitness and Fun (SCFFF), a 16-week community-based obesity prevention program, on children's moderate to vigorous physical activity (MVPA), physical fitness, body mass index (BMI), and BMI z-score. A non-randomized pre-test and post-test study design was used to assess changes in MVPA, physical fitness, BMI and BMI z-score from baseline to program end. MVPA was measured by accelerometer, physical fitness measured by Fitnessgram, and height and weight were measured by stadiometer and scale and used to calculate BMI. The analytic sample for this study included 53 children from nine SCFFF programs conducted between 2011-2016. There was a significant increase in the percentage of time children spent participating in MVPA $(1.12 \%$; $\mathrm{p}=0.022)$, curl-ups $(\mathrm{p}<0.001)$ and trunk lifts $(p=0.004)$. BMI $z$-score decreased $(p<0.001)$. Results reinforce the importance of offering community-based interventions that include caregivers to prevent excess weight gain in children.
\end{abstract}

Keywords: children, physical activity, physical fitness, obesity, overweight, community-based intervention

Cite This Article: Furong Xu, Mary L. Greaney, Stephanie Marchand, Celeste Corcoran, Heather Di Biasio, Jane Baruch, Deborah Riebe, and Geoffrey W. Greene, "The Impact of a Community-Based Childhood Obesity Prevention Program on Children's Physical Activity and Fitness.” Journal of Physical Activity Research, vol. 2, no. 1 (2017): 44-49. doi: 10.12691/jpar-2-1-8.

\section{Introduction}

Childhood obesity is a global epidemic with nearly one of three children being classified as overweight or obese [1]. Lack of physical activity along with an unhealthful diet behavior contribute to childhood obesity [2], and are associated with cardiovascular disease risk factors and type II diabetes [3,4]. Physical activity plays a crucial role in preventing obesity as it helps with dynamic energy balance [2]. It is recommended that children get at least 60 minutes of moderate to vigorous intensity physical activity (MVPA) every day [5], but only $42 \%$ of children in the United States between the ages of $6-11$ and $25 \%$ of children ages 12-15 years meet this recommendation [6,7]. Children with obesity are less likely to meet physical activity guidelines compared to children who have a healthy weight and girls are less active than boys [6,7]. Fakhouri et al found that $29.5 \%$ of boys and $24.1 \%$ of girls at a healthy weight met current physical activity recommendation in contrast to $18 \%$ of boys and $19.6 \%$ of girls with obesity [7].
Community-based interventions can be effective in increasing physical activity and improving dietary intake among children [8,9,10,11]. However, only a limited number have included both children and their caregivers [9]. Including caregivers is important as they are instrumental in shaping children's diet and physical activity behaviors through modeling and providing access to physical activity and healthy eating opportunities [8,10,11,12]. Moreover, community-based obesity prevention programs often rely on self-reported measures of physical activity to determine intervention efficacy $[9,13,14]$. Given the importance of increasing physical activity, it is crucial to use objective measures to determine if offered community-based interventions are efficacious as self-reported measures overestimate children's physical activity $[9,10,15]$. Thus, the primary aim of the study is to determine effects of a community-based intervention designed for children and their caregivers on children's objectively measured physical activity and physical fitness. The secondary aim is to examine the intervention's effects on children's body mass index (BMI) z-scores and to investigate the association between change in MVPA and BMI z-scores. 


\section{Methods}

\subsection{Study Design and Participants}

A non-randomized pre-test and post-test study design was used to evaluate the impact of a 16-week communitybased intervention - South County Food Fitness and Fun (SCFFF) on physical activity, physical fitness, BMI, and BMI z-scores among pre-adolescent children enrolled in the program. The program is designed to be interactive thus only a limited number of children and their caregivers are enrolled in each session. The program was created in 2009 with support from South County Hospital in response to an identified need [16]. The SCFFF was collaboratively designed and implemented by local pediatricians, pediatric dietitians, community-based child activity specialists and university faculty and is offered once or twice a year at no cost. Children and their caregivers were referred to SCFFF by physicians from health care practices located in southern Rhode Island. Children were eligible to attend SCFFF if they were overweight (85th percentile $\leq \mathrm{BMI}<95$ th percentile) or obese (BMI $\geq 95$ th percentile) and between 6-10 years of age [17].

The analytic sample for the present study included children with complete accelerometer data who enrolled in one of nine SCFFF programs implemented between 2011 and 2016. The study was approved by the Institutional Review Board of the University of Rhode Island. Before the start of each SCFFF program, caregivers provided informed consent for themselves and their children and children signed assent forms.

\subsection{Intervention}

The SCFFF (see Table 1 for session outlines) is a 16-week community-based intervention designed for caregivers and children. Sessions are held once a week for 75 minutes at a local children's exercise studio. During the first 30 minutes of sessions 2-15, children participate in a nutrition session while the caregivers attend an exercise education session. After this, caregivers meet for a 30minute nutrition education session while children participated in supervised physical activity. The final 15 minutes of each session are devoted to an interactive physical activity session. The same pediatric dietitians and child activity specialist oversee the nutrition and physical activity sessions respectively (see Table 1 ).

The supervised physical activity sessions are geared towards fun activities that require minimal equipment and space so families could sustain the activity upon program completion of the program. Children participate in active games designed to help them develop lifetime skills. The interactive physical activity discussions with caregivers are designed to facilitate social support and to help caregivers develop strategies to help their children be more physically active. Each session closes with children and caregivers participating in 15 minutes of physical activity to encourage regular physical activity for the family. Families also receive a weekly physical activity challenge to encourage caregivers to exercise daily with their children outside of the SCFFF sessions.

The Children's nutrition education component emphasizes the importance of healthy eating through the use of interactive games and contests while the caregivers' sessions use an open forum to facilitate questions and share ideas for overcoming barriers to healthful eating. The nutrition sessions are designed to help caregivers get the skills self-efficacy to modify the home nutrition environment to promote healthy eating for the entire family. Children and their caregivers also participate in weekly nutrition challenges that reinforce learning and promote healthful eating.

Table 1. Description of the SCFFF program

\begin{tabular}{|c|c|c|}
\hline Audience & Nutrition Segment (30 minutes) & Physical Activity Segment (30 minutes) \\
\hline Child & $\begin{array}{l}\text { 1. Games focusing on Food Guide Pyramid/Choose MyPlate } \\
\text { food groups and serving sizes (Topic of the week) } \\
\text { 2. Food tasting games } \\
\text { 3. Importance of fruits, vegetables and whole grains } \\
\text { 4. Awareness of foods high in added sugar, sodium and fats } \\
\text { 5. Snacks versus treats } \\
\text { 6. Meals, fast foods and beverages } \\
\text { 7. Weekly Nutrition Challenge: Making recipe with } \\
\text { caregiver or art \& crafts related to topic of the week }\end{array}$ & $\begin{array}{l}\text { 1. Description and demonstration of target activity } \\
\text { 2. Weekly activity (rope jumping, ball playing, team games, } \\
\text { yoga, hand-eye coordination) } \\
\text { 3. Weekly activity challenge }\end{array}$ \\
\hline \multirow{10}{*}{ Caregivers } & $\begin{array}{l}\text { 1. Review previous week's Nutrition Challenge and recipe } \\
\text { tasting }\end{array}$ & \\
\hline & $\begin{array}{l}\text { 2. Food Guide Pyramid/Choose MyPlate food groups and } \\
\text { serving sizes }\end{array}$ & $\begin{array}{l}\text { 1. Review previous week's physical activity challenge, address } \\
\text { barriers and suggest resolutions for being physically active }\end{array}$ \\
\hline & 3. Foods high in added sugar, fats, sodium & 2. Types of physical activities and activity intensity levels \\
\hline & 4. Snacks versus treats & 3. Importance of physical activity for health \\
\hline & 5. Meals, fast foods and beverages & 4. Increasing physical activity in daily life using available \\
\hline & 6. Changing eating behaviors as a family & neighborhood resources (e.g., bike paths) \\
\hline & 7. Importance of fruits, vegetables and whole grains & 5. Parents as role models for active living \\
\hline & 8. Challenges in changing a child's eating behavior & 6. Family weekly physical activity challenge \\
\hline & 9. Caregivers as role models for healthful eating & \\
\hline & 10. Weekly Nutrition Challenge recipe & \\
\hline \multirow{3}{*}{ Child and Caregiver } & & Interactive physical activity (15 minutes) \\
\hline & & 1. Child teaches caregiver how to do activity \\
\hline & & 2. Joint activity based on weekly targeted physical activity \\
\hline
\end{tabular}

Note: SCFFF $=$ South County Food Fitness and Fun. 


\subsection{Measures}

All assessments were completed at a local children's exercise studio and during the first and last weeks of the SCFFF intervention (weeks 1 and 16).

\subsubsection{Demographic and Anthropometric Information}

Caregivers completed a baseline survey where they reported their child's sex and age. A physician affiliated with SCFFF measured children's height and weight at baseline and at the end of the program. Height was measured to the closest 0.25 inches using a stadiometer (model WB3000, Arlington Heights, Illinois). Weight was calculated to the closest 0.1 pound using a balance beam scale (model WB300731, Arlington Heights, Illinois). Height and weight were used to calculate BMI, and age and sex-specific BMI z-scores using the Children's Hospital of Philadelphia Research Institute calculator [18].

\subsubsection{Physical Activity Levels}

Children's physical activity was measured using an ActiGraph GT3X accelerometer (Pensacola, FL), a valid tool to assess children's physical activity volume and intensity $[19,20]$. Accelerometers were initialized using 60-s epoch [21]. Children were asked to wear the accelerometer for seven days during the first and last weeks of the program and to wear the accelerometer on their left anterior mid-line of the thigh around the waist during waking hours and only remove it during water activities such as swimming and showering [20]. A non-wear period was defined as 60 minutes of consecutive zeros [22]. Valid accelerometer wear time consisted of a minimum of 3 days and 6 hours per day with at least one weekend day [23]. Physical activity in minutes was determined using Mackintosh et al.'s accelerometer calibration cut points for children in free-living situation and MVPA was defined as $>2160$ counts $\bullet \min ^{-1}$ [24]. In addition, accelerometer data was used to determine average duration of each bout of MVPA [25]. As there are no established guidelines for children's MVPA bout duration [26], we defined a MVPA bout as physical activity that was maintained $>2160$ counts $\bullet \mathrm{min}^{-1}$ for $\geq 1$ minute as children perform physical activity primarily in sporadic bursts [25]. Percentage measured wear time in MVPA was defined as (MVPA min/valid wear time) $\mathrm{x}$ 100. MVPA minutes and bouts were averaged from days with valid accelerometer time. Duration of bouts and percentage of measured wear time in MVPA was averaged using valid wear time.

\subsubsection{Physical Fitness}

Health-related physical fitness was assessed using measures from the Fitnessgram that assess muscular strength and endurance (push-ups; curl-up), Trunk extensor strength and flexibility (trunk lifts) and flexibility (sit and reach; shoulder stretch) (Cooper Institute, Dallas, Texas) [27]. Standardized protocols were used for the assessments that were scored using age and gender specific criterion standards [27]. The Fitnessgram is a validated and reliable evaluation for children between the ages of 5-18 years old [28].

\subsection{Statistical Analysis}

Baseline differences between participants with complete accelerometer data at both time points and those missing post data were assessed using independent $t$ tests or Chi-square tests. To assess change from pre-to post, paired t-test were conducted for normally distributed continuous variables, Wilcoxon signed rank test were employed for variables that were not normally distributed, and Chisquare test and McNemar's tests were used to assess change for categorical variables. Effect size was used to quantify the effect of the intervention on MVPA and other measures calculated using Cohen's d or risk difference. Analysis of covariance was used to compare differences in MVPA at the end of the program adjusting for baseline scores and weekdays and weekends due to differences in MVPA noted by previous studies [7,29]. The correlations between MVPA and the physical fitness measures as well as relative weight were determined by Person product-moment correlation. All analyses were conducted using the SPSS (version 22; IBM Corp., Armonk, NY), and statistical significance was set at $\mathrm{p}<0.05$.

\section{Results}

Fifty-three (72.6\%) of 73 children who enrolled in SCFFF attended at least 11 of the 16 sessions and were classified as completers. Baseline characteristics among completers and non-completers are summarized in Table 2. Forty-nine $\left(\mathrm{n}=49,92 \%\right.$; $\mathrm{BMI}=24.8 \pm 4.8 \mathrm{~kg} / \mathrm{m}^{2}$, age $=8.5 \pm$ $1.3 \mathrm{yrs}$ ) of the 53 completers met accelerometer protocols at baseline, but only 40 (76\%) had valid wear time at both time points. Only a small proportion (4.7\%) of children's average daily wear time at baseline (622 minutes/day) was spent in MVPA (mean = 29.9 minutes, $\mathrm{SD}=13.7$ minutes) of MVPA per day. Only one child (2\%) met the recommended 60 minutes of MVPA per day at baseline assessment. Accumulated MVPA consisted of an average of 1.8 minutes per bout duration with average of 16.5 MVPA bouts per day. Children without valid wear time at post $(n=9)$ had significantly lower MVPA time and minutes of wear time at baseline than those with valid wear time at both time points (Table 2).

For children with valid wear time at both time points ( $n=40), 63 \%$ (25 of 40) participants had an average of 5.7 days of valid wear time at baseline, compared to $45 \%$ (18 of 40) with average of 4.95 days of valid wear time at post. As shown in Table 3, the proportion of children's daily MVPA time increased significantly from baseline to post $(p=0.02, d=0.43)$ despite wear time decreasing from baseline to post ( $\mathrm{p}=0.025, d=-0.46$ ). There was no change in overall measured daily MVPA minutes, in the number of daily MVPA bouts or the average duration per bout. In addition, there were no differences in daily MVPA or percent daily time spent in MVPA by sex.

At the end of the intervention, there was a significant increase in muscular strength and endurance as well as trunk extensor strength and flexibility as assessed by the number of curl-ups and trunk lifts (Table 4). The increase in number of curl-up was inversely correlated with changes in BMI ( $\mathrm{r}=-0.42, \mathrm{p}=0.07)$ and BMI $\mathrm{z}$-scores 
( $r=-0.47, p=0.003$ ). Change in trunk lifts was related to change in percent daily time spent in MVPA ( $\mathrm{r}=0.33$, $\mathrm{p}=0.038$ ). There were no observed differences by sex in the five fitness subtests between the two time points, although boys had significant improvements in curl ups (mean difference 5.14, 95\% CI 0.85 to 9.43; $\mathrm{p}=0.022$ ) and sit and reach (mean difference $1.39 \mathrm{~cm}, 95 \%$ CI 0.49 to $2.29 \mathrm{~cm} ; \mathrm{p}=0.005)$. Girls demonstrated significant improvements in curl ups (mean difference 6.36, 95\% CI
3.23 to $9.49 ; \mathrm{p}<0.001$ ) and trunk lifts (mean difference 1.08 , 95\% CI 0.16 to 2.00 ; $\mathrm{p}=0.015$ ).

BMI and BMI z-scores decreased over the course of the intervention (see Table 4) while weight remained stable (mean difference 1.29 , 95\% CI -0.52 to $3.11 ; \mathrm{p}=0.156$ ) and height increased (54.6 \pm 3.85 inches to $55.4 \pm 3.85$ inches, $\mathrm{p}<0.01$ ). Exploratory analyses revealed that there were no observed sex differences in BMI or BMI z-scores. There was no association between change in BMI $\mathrm{z}$ and change in MVPA.

Table 2. Baseline characteristics of SCFFF completers $(n=49)^{\mathrm{a}}$

\begin{tabular}{|c|c|c|c|c|}
\hline Variables & Total $(n=49)$ & $\begin{array}{l}\text { Completers with valid wear time at } \\
\text { baseline and post }(n=40)\end{array}$ & $\begin{array}{l}\text { Completers with valid wear } \\
\text { time only at baseline }(n=9)\end{array}$ & p-value \\
\hline Age (years) & $8.47 \pm 1.29$ & $8.63 \pm 1.27$ & $7.78 \pm 1.20$ & 0.075 \\
\hline \multicolumn{5}{|l|}{ Weight related measures } \\
\hline BMI $\left(\mathrm{kg} / \mathrm{m}^{2}\right)$ & $24.83 \pm 4.75$ & $24.99 \pm 4.93$ & $24.14 \pm 4.04$ & 0.637 \\
\hline BMI z-score & $2.03 \pm 0.53$ & $2.03 \pm 0.50$ & $2.02 \pm 0.69$ & 0.937 \\
\hline \multicolumn{5}{|l|}{ Physical activity } \\
\hline Daily MVPA (min) & $29.99 \pm 13.70$ & $32.04 \pm 13.11$ & $20.88 \pm 13.21$ & $0.026^{\mathrm{b}}$ \\
\hline Daily MVPA bouts (frequency) & $16.45 \pm 7.47$ & $17.70 \pm 7.27$ & $10.90 \pm 5.92$ & $0.012^{\mathrm{b}}$ \\
\hline Duration per MVPA bout (min) & $1.82 \pm 0.36$ & $1.82 \pm 0.34$ & $1.79 \pm 0.46$ & 0.872 \\
\hline Daily wear time (min) & $621.96 \pm 148.31$ & $652.15 \pm 119.40$ & $487.78 \pm 194.06$ & $0.002^{\mathrm{b}}$ \\
\hline Daily proportion of time as MVPA (\%) & $4.72 \pm 2.00$ & $4.93 \pm 1.87$ & $3.79 \pm 2.38$ & 0.121 \\
\hline \multicolumn{5}{|l|}{ Physical fitness } \\
\hline Curl up (number) & $5.63 \pm 6.86$ & $5.30 \pm 5.69$ & $7.11 \pm 11.02$ & 0.480 \\
\hline Trunk lift (cm) & $6.76 \pm 2.94$ & $6.41 \pm 2.99$ & $8.28 \pm 2.31$ & 0.086 \\
\hline Push up (number) & $4.45 \pm 4.57$ & $4.78 \pm 4.83$ & $3.00 \pm 2.92$ & 0.297 \\
\hline Sit and reach $(\mathrm{R})(\mathrm{cm})$ & $-1.20 \pm 3.17$ & $-1.18 \pm 3.08$ & $-1.33 \pm 3.74$ & 0.894 \\
\hline Sit and reach $(\mathrm{L})(\mathrm{cm})$ & $-1.23 \pm 2.95$ & $-1.21 \pm 2.77$ & $-1.33 \pm 3.87$ & 0.913 \\
\hline Shoulder stretch (R) n (\%) ${ }^{\mathrm{c}}$ & $28(57.14)$ & $23(57.50)$ & $5(55.56)$ & 0.711 \\
\hline Shoulder stretch (L) n (\%) ${ }^{c}$ & $27(55.10)$ & $22(55.00)$ & $5(55.56)$ & 0.669 \\
\hline
\end{tabular}

Note: completers are children who attended at least 2/3 of the sessions. SCFFF = South County Food Fitness and Fun. Values were mean \pm SD unless otherwise specified. ${ }^{a}$ four completers were excluded because they did not meet the criterion for valid wear time. ${ }^{\mathrm{b}} \mathrm{p} \leq 0.05$. ${ }^{\mathrm{c}} \mathrm{p}$-value was obtained by performing Chi-square test. Other p-values were obtained by performing t-test or Wilcoxon Mann Whitney test.

Table 3. Comparison of post and pre- physical activity measures in SCFFF program (n=40)

\begin{tabular}{|c|c|c|c|c|c|}
\hline Variables & Duration & Pre- & Post- & Effect size (95\%CI) & p-value \\
\hline Daily MVPA (min) & weekly & $32.04 \pm 13.11$ & $32.53 \pm 13.12$ & $0.04(-3.36-4.34)$ & 0.799 \\
\hline Daily MVPA bouts (frequency) & weekly & $17.70 \pm 7.27$ & $17.98 \pm 6.61$ & $0.04(-1.88-2.44)$ & 0.795 \\
\hline Duration per MVPA bout (min) & weekly & $1.82 \pm 0.340$ & $1.81 \pm 0.41$ & $-0.03(-0.12-0.11)$ & 0.879 \\
\hline Daily wear time (min) & weekly & $652.15 \pm 119.40$ & $587.53 \pm 159.96$ & $-0.46(-120.65--8.59)$ & $0.025^{\mathrm{a}}$ \\
\hline Daily proportion of time as MVPA (\%) & weekly & $4.93 \pm 1.87$ & $6.05 \pm 3.22$ & $0.43(0.17-2.07)$ & $0.022^{\mathrm{a}}$ \\
\hline Daily MVPA (min) & weekdays & $29.35 \pm 14.06$ & $31.04 \pm 12.75$ & $0.13(-2.38-5.77)$ & 0.406 \\
\hline Daily wear time (min) & weekdays & $680.35 \pm 128.52$ & $636.43 \pm 141.73$ & $-0.32(-99.14-11.31)$ & 0.116 \\
\hline Daily proportion of time as MVPA (\%) & weekdays & $4.36 \pm 1.93$ & $5.11 \pm 2.38$ & $0.35(0.04-1.46)$ & $0.040^{\mathrm{a}}$ \\
\hline Daily MVPA(min) & weekend & $40.22 \pm 24.40$ & $38.74 \pm 22.15$ & $-0.06(-11.03-8.06)$ & 0.752 \\
\hline Daily wear time(min) & weekend & $581.15 \pm 193.34$ & $462.34 \pm 314.05$ & $-0.46(-232.47--5.16)$ & $0.041^{\mathrm{a}}$ \\
\hline Daily proportion of time as MVPA (\%) & weekend & $6.60 \pm 6.98$ & $8.25 \pm 7.46$ & $-0.23(-1.99-5.30)$ & 0.357 \\
\hline
\end{tabular}

Note: SCFFF $=$ South County Food Fitness and Fun. Values were mean \pm SD unless otherwise specified. MVPA refers to minutes of moderate-tovigorous physical activity. ${ }^{\mathrm{a}} \mathrm{p} \leq 0.05$.

Table 4. Comparison of SCFFF post- and pre- weight related and physical fitness measures (n=40)

\begin{tabular}{lccc}
\hline Variables & Pre- & Post- & Effect size (95\%CI) \\
\hline Weight related measures & & & \\
BMI $\left(\mathrm{kg} / \mathrm{m}^{2}\right)$ & $24.9 \pm 4.99$ & $24.25 \pm 4.16$ & $-0.14(-1.36--0.09)$ \\
BMI z-score & $2.0 \pm 0.51$ & $1.92 \pm 0.53$ & $-0.16(-0.17--0.06)$ \\
Physical fitness & & & \\
Curl up & $5.30 \pm 5.69$ & $11.21 \pm 7.11$ & $0.92(3.51-8.34)$ \\
Trunk lift (cm) & $6.41 \pm 2.99$ & $7.47 \pm 3.45$ & $0.33(0.39-1.97)$ \\
Push up & $4.78 \pm 4.83$ & $5.04 \pm 4.44$ & $0.06(-0.84-1.48)$ \\
Sit and reach (R) (cm) & $-1.18 \pm 3.08$ & $-0.55 \pm 2.58$ & $0.22(-0.03-1.49)$ \\
Sit and reach (L)(cm) & $-1.21 \pm 2.77$ & $-1.05 \pm 2.79$ & $0.06(-0.62-1.11)$ \\
Shoulder stretch (R) n (\%) & $23(57.50)$ & $24(59.5)$ & $0.02(-0.20-0.23)^{\mathrm{c}}$ \\
Shoulder stretch (L) n (\%) & $22(55.00)$ & $20(50.0)$ & $0.001^{\mathrm{a}}$ \\
\hline
\end{tabular}

Note: SCFFF $=$ South County Food Fitness and Fun. Values were mean \pm SD unless otherwise specified. MVPA refers to minutes of moderate-tovigorous physical activity. ${ }^{\mathrm{a}} \mathrm{p} \leq 0.05 .{ }^{\mathrm{b}} \mathrm{p}$-values were obtained by performing McNemar's Test. ${ }^{\mathrm{c}}$ risk difference was calculated for effect size. 


\section{Discussion}

This study evaluated the effectiveness of SCFFF, a 16week community-based intervention that included children and their caregivers, on children's physical activity, physical fitness and weight status. Children who attended SCFFF maintained minutes of MVPA but increased the percentage of time they spent participating in MVPA. Furthermore, they increased muscle strength and endurance and trunk extensor strength and flexibility which was noted by improved in the number of trunk lift and curl up but their BMI z-scores decreased.

Among SCFFF completers, minutes of MVPA remained stable despite valid accelerometer wear time significantly decreased from baseline to post. Robertson and colleagues (2010) also found that daily MVPA time remained stable over the course of the intervention but their study shown more consistent accelerometer wear time at both time points [30]. Additionally, the proportion of completers in this study with valid wear time (76\%) is lower than $90 \%$ in Robertson and colleagues' study [30], but similar to $71 \%$ reported by the National Health and Nutrition Examination Survey [6], indicating that compliance with prescribed wear time among children is a challenging, although not uncommon issue [31,32]. The seven-day measurement time period was used for the present study as it provides reliable $(\mathrm{R}>0.76)$ estimation of children' physical activity levels [33] but this was difficult to implement as only $63 \%$ of children had close to 6 days at baseline and $45 \%$ had close to 5 days of valid wear time at follow up. A large-scale study found that there was 15\% reduction from baseline to follow-up in the number of participants having 5 valid days of accelerometer wear time following a 20-month intervention [34]. Children may not want wear accelerometers due to negative peer pressure, forgetfulness, or discomfort [35]. The nine children in the present study who did not have a minimum of 3 days of valid wear time at follow-up had lower wear time as well as MVPA at baseline in contrast to completers with valid wear time at both time points, which suggests that less active children were less compliant with accelerometer protocols.

There was moderate intervention effect $(d=0.4)$ in the percentage of time spent in MVPA (1.1\%), which is comparable to previous studies [36,37]. However, it is difficult to directly compare this study with others due to use of different cut points for MVPA [37] and devices [36]. Children participating in SCFFF accumulated MVPA in sporadic bouts [24], with an average bout of less than 2 minutes. To the best of our knowledge, this is the first community-based obesity intervention study reporting accumulation of MVPA bouts. Thus, future research is needed to investigate its impact on MVPA bouts and association between MVPA bouts and weight status in community-based childhood obesity interventions.

Children completing SCFFF increased their scores in trunk lifts and curl ups both of which are important for overall physical function [38], and the intervention effect for curl up was large $(d=0.9)$. Other community-based studies report similar results $[39,40]$. The SCFFF intervention was designed to promote daily lifestyle physical activity through fun activities which could be sustained at home with parental support. As a result of that, it is possible that activities that children were chosen only benefit particular components of fitness (e.g., curl ups) not others (e.g., push up). Furthermore, the change in trunk lift explained 11\% of the variance in percent change in daily MVPA $(r=0.33$, $\mathrm{p}=0.038$ ). This finding indicates that children who improved their strength and endurance may also have increased their MVPA since more physical activity participation lead to improving physical fitness [39].

Participation in SCFFF may have contributed to reduced BMI z-score. Other studies also have found BMI z-score decreased after participation in community-based physical activity and nutrition interventions [31,37]. We did not find a relationship between changes in MVPA and changes in BMI z-scores which may be due to the relatively small changes in MVPA and to the relatively short duration of SCFFF intervention. The extant literature shows mixed results about the relationship between changes in MVPA and changes BMI z-scores [29,31,37,40]. Weight change is a complex issue influenced other factors such as nutrition [37].

The current study should be considered in light of its strengths and limitations. The SCFFF was a communityengaged research which was a collaborative effort among community stakeholders including local pediatricians, pediatric dietitians, community-based child activity specialists and university faculty. The interactive nature of the SCFFF intervention and the inclusion of caregivers were strengths of the intervention [8]. The use of accelerometer is a strength of this study and it provides an objective measure of physical activity. Although compliance with accelerometer protocols is a limitation of the study, issues with compliance is common in children and in childhood obesity interventions [30,32]. Another limitation is that this study did not assess caregivers' physical activity behaviors, BMI and BMI z-score. The present study also has limited generalizability as the participants were primarily white living in a rural/suburban area thus findings may not be applied to differing populations.

\section{Conclusion}

Our study showed that SCFFF was effective at improving percent of time in MVPA, improving children's muscle strength and endurance and trunk extensor strength and flexibility, and decreasing children's BMI z-scores. Further research is needed using more diverse participants, and an intervention that utilizes a control group. Standardized accelerometer calibration cut points for children are also needed and use of a more attractive, waterproof accelerometer may increase compliance. This study contributes to existing literature and demonstrates the effectiveness of a childhood obesity intervention program on making positive lifestyle changes in overweight and obese children.

\section{Acknowledgements}

The authors thank all program participants and their caretakers/guardians for participating in South Count Food Fitness and Fun (SCFFF). We also would like to thank all student volunteers and community members who have donated their time and expertise to support SCFFF, 
and Rachel Clough, an exercise specialist, for her assistance and contribution to SCFFF.

\section{Competing Interests}

The authors declared no competing interest regarding the study results or the publication of this paper.

\section{References}

[1] Ogden CL, Carroll MD, Kit BK, Flegal KM. Prevalence of obesity and trends in body mass index among US children and adolescents, 1999-2010. JAMA. 2012; 307(5): 483-490.

[2] Hall KD, Heymsfield SB, Kemnitz J, Klein S, Schoeller DA. Speakman JR. Energy balance and its components: Implications for body weight regulation. Am. J. Clin. Nutr. 2012; 95(4): 989-994.

[3] Durstine JL, Gordon B, Wang Z, Luo X. Chronic disease and the link to physical activity. Journal of Sport and Health Science. 2013: 2(1): 3-11.

[4] Freedman DS, Mei Z, Srinivasan SR, Berenson GS, Dietz WH. Cardiovascular risk factors and excess adiposity among overweight children and adolescents: The Bogalusa Heart Study. J Pediatr. 2007; 150: 12-17.

[5] US Department of Health and Human Services. Physical activity guidelines for Americans. Washington, DC: US Department of Health and Human Services; 2008.

[6] Troiano RP, Berrigan D, Dodd KW, Masse LC, Tilert T, Mcdowell M. Physical activity in the United States measured by accelerometer. Med Sci Sports Exerc. 2008; 40: 181-188.

[7] Fakhouri TH, Hughes JP, Burt VL, Song M, Fulton JE, Ogden CL. Physical activity in US youth aged 12-15 years. 2012 NCHS Dato Brief. 2014; 141: 1-8.

[8] Anderson JD, Newby R, Kehm R, Barland P, Hearst MO. Taking steps together: a family- and community-based obesity intervention for urban, multiethnic children. Health Educ Behav. 2015; 42(2): 194-201.

[9] Ickes MJ, Sharma M. A systematic review of community-based childhood obesity prevention programs. J Obes Weight Loss Ther. 2013; 3: 188

[10] Robertson W, Thorogood M, Inglis N, Grainger C, Stewart-Brown S. Two-year follow-up of the 'Families for Health' programme for the treatment of childhood obesity. Child Care Health Dev. 2011; 38 (2): 229-236.

[11] Sacher PM, Kolotouru M, Chadwick PM, Cole TJ, Lawson MS, Lucas A, et al. Randomized controlled trial of the MEND program: a family-based community intervention for childhood obesity. Obesity (Silver Spring). 2010; 18 Suppl 1: S62-68.

[12] Bandura A. Social Foundations for Thought and Action: A Social Cognitive Theory. Englewood Cliffs, NJ, Prentice Hall; 1986.

[13] Black MM, Hager ER, Le K, Anliker J, Arteaga SS, Diclemente C, et al., Challenge! Health promotion/obesity prevention mentorship model among urban, black adolescents. Pediatrics. 2010; 126(2): 280-288.

[14] Hardman CA, Horne PJ, Lowe F. A home-based intervention to increase physical activity in girls: The fit "n" fun dudes program. $J$ Ex Sci Fitness. 2009; 7: 1-8.

[15] Pereira S, Gomes TN, Borges A. Variability and stability in daily moderate-to-vigorous physical activity among 10-year-old children. Int J Environ Res Public Health. 2015; 12: 9248-9263.

[16] Bryant E B. Rhode Island KIDS COUNT Factbook, 1997, 1997.

[17] Cole TJ, Bellizzi MC, Flegal KM, Dietz WH. Establishing a standard definition for child overweight and obesity worldwide: international survey. BMJ. 2000; 320: 1240-1245.

[18] Children's Hospital of Philadelphia Research Institute. Pediatric Z-score Calculator. Available from: http://zscore.research.chop.edu.

[19] Pate RR, Almeida MJ, McIver KL, Pfeiffer KA, Dowda M. Validation and calibration of an accelerometer in preschool children. Obesity (Silver Spring). 2006; 14(11): 2000-2006.
[20] Rowlands AV, Rennie K, Kozarski R. Children’s physical activity assessed with hip- and wrist-worn accelerometers. Med Sci Sports Exerc. 2014; 46(12): 2308-2316.

[21] Pate RR. Measuring physical activity. In: Ward DS, Saunders RP, Pate RR, editors. Physical activity interventions in children and adolescents. Champaign (IL): Human Kinetics; 2007. p. 145-65.

[22] Janssen X, Basterfield L, Parkinson KN, Pearce MS, Reilly JK, Adamson AJ, et al. Objective measurement of sedentary behavior: impact of non-wear time rules on changes in sedentary time. BMC Public Health. 2015; 15: 504.

[23] Schaefer CA, Nigg CR, Hill JO, Brink LA, Browning RC. Establishing and evaluating wrist cutpoints for the GENEActiv accelerometer in youth. Med Sci Sports Exerc. 2014; 46(4): 826-833.

[24] Mattocks C, Ness A, Leary S, Leary SD, Tilling K, Blair SN. Use of accelerometers in a large field-based study of children: protocols, design issues, and effects on precision. $J$ Phys Act Health. 2008; 5 Suppl 11: S98-S111.

[25] Mackintosh KA, Fairclough SJ, Stratton G, Ridgers ND. A calibration protocol for population-specific accelerometer cutpoints in children. PLoS One. 2012; 7: e36919.

[26] Masse LC, Fuemmeler BF, Anderson CB, Matthews CE, Trost SG Catellier DJ, et al. Accelerometer data reduction: a comparison of four reduction algorithms on select outcome variables. Med Sci Sports Exerc. 2015; 37(11): S544-S554.

[27] Plowman SA, Meredith MD. Fitnessgram/Activitygram Reference Guide (4th Eds.). Dallas, TX, The Cooper Institute; 2013.

[28] Morrow JR, Martin SB, Jackson AW. Reliability and validity of the FITNESSGRAM. Res Q Exerc Sport. 2010; 81(3): S24-S30.

[29] Carson V, Stone M, Faulkner G. Patterns of sedentary behavior and weight status among children. Pediatr. Exerc. Sci. 2014; 26(1): 95-102.

[30] Robertson W, Stewart-Brown S, Wilcock E, Oldfield M, Thorogood M. Utility of Accelerometers to Measure Physical Activity in Children Attending an Obesity Treatment Intervention. Journal of Obesity. 2011; 2011: 398918.

[31] Basterfield L, Jones AR, Parkinson KN, Reilly J, Pearce MS, Reilly JJ. Physical activity, diet and BMI in children aged 6-8 years: a cross-sectional analysis. BMJ Open. 2014; 4 (6): e005001.

[32] Wells SL, Kipping RR, Jago R, Brown J, Hucker D, Blackett A. Characteristics associated with requested and required accelerometer wear in children. BMJ Open. 2013; 3(8): e003402.

[33] Trost SG, Pate RR, Freedson PS, Sallis JF, Taylor WC. Using objective physical activity measures with youth: how many days of monitoring are needed? Med. Sci. Sports Exerc. 2000; 32: 426-431.

[34] Grydeland M, Bergh IH, Bjelland M, et al. Intervention effects on physical activity: the HEIA study - a cluster randomized controlled trial. The International Journal of Behavioral Nutrition and Physical Activity. 2013; 10:17.

[35] Kirby J, Tibbins C, Callens C, et al. Young people's views on accelerometer use in physical activity research: findings from a user involvement investigation. ISRN Obesity. 2012; 2012:948504.

[36] Goran MI, Reynolds K. Interactive Multimedia for Promoting Physical Activity (IMPACT) in children. Obesity Research. 2005; 13:762-771.

[37] Trinh A, Campbell M, Ukoumunne OC, Gerner B, Wake M. Physical activity and 3-year BMI change in overweight and obese children. Pediatrics. 2013; 131(2): e470-e477.

[38] Malina RM, Bouchard C, Bar-Or O. Growth, maturation, and physical activity. 2nd. Champaign, IL: Human Kinetics Publishers; 2004.

[39] Rauner A, Mess F, Woll A. The relationship between physical activity, physical fitness and overweight in adolescents: a systematic review of studies published in or after 2000. BMC Pediatr. 2013; 13(1): 19.

[40] Willis EA, Ptomey LT, Szabo-Reed AN, Honas JJ, Lee J, Washburn RA, et al. Length of moderate-to-vigorous physical activity bouts and cardio-metabolic risk factors in elementary school children. Preventive Medicine. 2015; 73: 76-80 